\title{
Mehr Geld für Heimbesuche
}

\author{
Ärzte sollen künftig für Heimbesuche besser bezahlt werden. Das sieht die Pflegereform von \\ Gesundheitsminister Daniel Bahr (FDP) vor, die das Kabinett Ende März in Berlin beschlossen hat. \\ Insgesamt sollen den Ärzten 77 Millionen € zusätzlich zur Verfügung stehen.
}

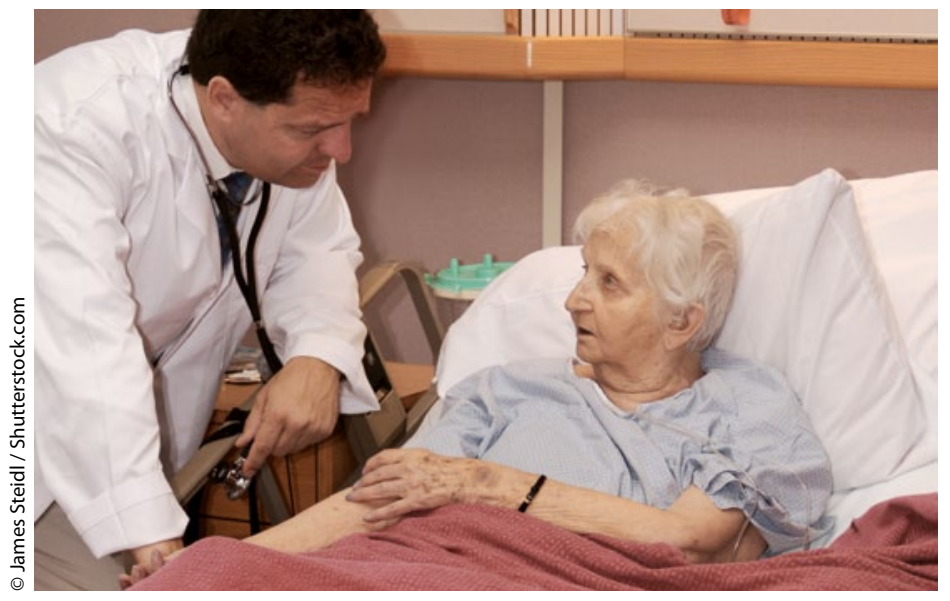

Nach der Pflegereform sollen Arztbesuche in Pflegeheimen besser bezahlt werden.

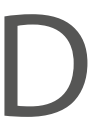
as zusätzliche Geld für Heimbesuche ist eine sinnvolle Investition", betonte Bahr anlässlich der Vorstellung seiner Pflegereform. Damit würde die medizinische Versorgung in Heimen verbessert werden. Ziel sei es, unnötige Krankenhauseinweisungen zu verhindern. Heimbewohner würden besonders am Wochenende häufig ins Kran- kenhaus gebracht. „Jeder Krankenhaustransport, der durch eine bessere medizinische Versorgung im Heim vermieden werden kann, spart Geld“, so Bahr. Nach einigen Jahren soll evaluiert werden, wie viel tatsächlich mit diesen Maßnahmen eingespart werden könne. Für die Umsetzung würden bereits Gespräche mit der Selbstverwaltung geführt.

\section{Mehr Geld auch für die Versorgung Demenzkranker zu Hause}

Auch Pflegebedürftige mit Demenz, die zu Hause versorgt werden, sollen ab 1 . Januar 2013 mehr Geld erhalten. Dafür soll der Beitrag zur sozialen Pflegeversicherung um 0,1 Prozentpunkte auf 2,05\% des gesetzlichen Bruttolohnes angehoben werden. Das soll jährlich 1,1 Milliarden $€$ einbringen.

Lob kam von der Kassenärztlichen Bundesvereinigung: „Es ist ein richtiger Gedanke, dass Ärzte für den enormen Arbeitseinsatz, den sie in Heimen leisten, auch mehr Geld erhalten sollen“, sagte KBV-Sprecher Roland Stahl.

Der GKV-Spitzenverband lehnte die neue Regelung ab: „Der Sicherstellungsauftrag in der ambulanten Versorgung gilt auch für Pflegebedürftige“, so Verbandssprecher Florian Lanz. Eine Ausnahme könne höchstens für Zahnärzte gelten, die gegebenenfalls besonderes technisches Gerät für Heimbesuche benötigten. Auch die Opposition kritisierte Bahrs Reform scharf.

\section{Rauchverbote sparen dem Gesundheitssystem Geld}

Weniger Angina Pectoris, sinkende Herzinfarktraten. Einer Hochrechnung der DAK Gesundheit zufolge sparen Rauchverbote der gesetzlichen Krankenversicherung 150 Millionen $€$ im Jahr. Diese Extrapolation wagte DAK GesundheitsChef Professor Herbert Rebscher bei der Vorstellung einer auf DAK-Daten beruhenden Studie am 13. März in Berlin. Im Jahr 2008, nach Einführung der Nichtraucherschutzgesetze in Deutschland, habe die Krankenkasse bei diesen Indikationen 1.880 Krankenhauseinweisungen weniger verzeichnet. Daraus errechneten sich
Minderausgaben von 7,7 Millionen $€$ bezogen auf eine Teilpopulation von 3,7 Millionen Versicherten der DAK. Die Zahlen müssten die Politiker in Bund und Ländern zur Kenntnis nehmen. „Der Diskurs über die Freiheitsrechte muss geführt werden", plädierte Rebscher für weitergehende Rauchverbote.

Die Vorher-Nachher-Studie „Nichtraucherschutzgesetze in Deutschland und

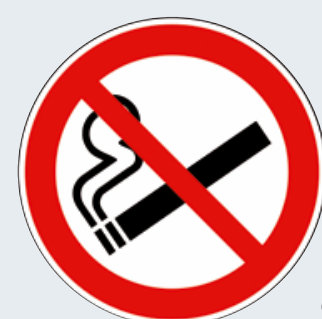

Krankenhausaufnahmen aufgrund von Angina Pectoris und akutem Herzinfarkt" betrachtet die Jahre 2004 bis 2008. Die Länder haben die Nichtraucherschutzgesetze zwischen August 2007 und Juli 2008 eingeführt. Damit hätten sie eine Trendumkehr bei den Krankenhausaufnahmen bei Angina Pectoris und Herzinfarkt eingeleitet, heißt es in der Studie. In den 36 Monaten vor Einführung der Gesetze waren die Einweisungen wegen Herzinfarkten um rund $3,5 \% \mathrm{im}$ Jahr gestiegen. 